\title{
Comparative effects of microvascular and macrovascular disease on the risk of major outcomes in patients with type 2 diabetes
}

Kamel Mohammedi ${ }^{1,2,3^{*}}$, Mark Woodward ${ }^{1,4,5}$, Michel Marre ${ }^{2,3,6}$, Stephen Colagiuri ${ }^{7}$, Mark Cooper ${ }^{8}$, Stephen Harrap ${ }^{9}$, Giuseppe Mancia ${ }^{10}$, Neil Poulter ${ }^{11}$, Bryan Williams ${ }^{12}$, Sophia Zoungas ${ }^{1,13}$ and John Chalmers ${ }^{1}$

\begin{abstract}
Background: Microvascular disease is associated with a high risk of macrovascular events in patients with type 2 diabetes, but the impact of macrovascular disease on the risk of microvascular events remains unknown. We sought to evaluate the respective effects of prior microvascular and macrovascular disease on the risk of major outcomes, including microvascular events, in these patients.
\end{abstract}

Methods: Participants in the Action in Diabetes and Vascular Disease: PreterAx and DiamicroN Modified-Release Controlled Evaluation (ADVANCE) trial $(n=11,140)$ and the ADVANCE-ON post-trial study $(n=8494)$ were categorized into 4 groups at baseline: dual absence of microvascular or macrovascular disease $(n=6789)$, presence of microvascular disease alone $(n=761)$, macrovascular disease alone $(n=3196)$, and both $(n=394)$. Outcomes were all-cause mortality, major macrovascular events (MACE), and major clinical microvascular events.

Results: All-cause mortality, MACE, and major clinical microvascular events occurred in 2265 (20\%), 2166 (19\%), and 807 (7\%) participants respectively, during a median follow-up of 9.9 (inter-quartile interval 5.6-10.9) years. The adjusted hazard ratios [95\% CI] of death, MACE, and major clinical microvascular events were each greater in patients with baseline microvascular disease (1.43 [1.20-1.71], 1.64 [1.37-1.97], and 4.74 [3.86-5.82], respectively), macrovascular disease (1.43 [1.30-1.57], 2.04 [1.86-2.25], and 1.26 [1.06-1.51]) or both (2.01 [1.65-2.45], 2.92 [2.40-3.55], and 6.30 [4.93-8.06]) compared with those without these conditions. No interaction was observed between baseline microvascular and macrovascular disease for these events. The addition of microvascular disease (change in c-statistic [95\% $\mathrm{Cl} 0.005$ [0.002-0.008], $\mathrm{p}=0.02)$ or macrovascular disease $(0.005$ [0.002-0.007], $\mathrm{p}<0.0001)$ considered separately or together (0.011 [0.007-0.014], $\mathrm{p}<0.0001$ ) improved the discrimination and the classification (integrated discrimination improvement (IDI): 0.013 [0.010-0.016], p < 0.001; net reclassification improvement (NRI): 0.021 [0.011-0.032], $p<0.001)$ of the risk of all-cause mortality. Microvascular disease improved discrimination (0.009 [0.003-0.014]) and classification (IDI: 0.008 [0.006-0.010]; NRI: 0.011 [0.001-0.020]) of MACE. Baseline macrovascular disease modestly enhanced IDI (0.002 [0.001-0.002]) and NRI (0.041 [0.002-0.087]), but not discrimination, of major clinical microvascular events.

Conclusions: Microvascular and macrovascular disease are independently associated with the 10-year risk of death, MACE, and major clinical microvascular events in patients with type 2 diabetes. The coexistence of these conditions was associated with the highest risks.

Keywords: Type 2 diabetes, Microvascular disease, Macrovascular disease, Mortality

\footnotetext{
*Correspondence: kmohammedi@georgeinstitute.org.au

${ }^{1}$ The George Institute for Global Health, University of Sydney, Sydney,

NSW, Australia

Full list of author information is available at the end of the article
} 


\section{Background}

Type 2 diabetes is a leading cause of microvascular complications and confers an excess risk of cardiovascular disease and death [1,2]. Microvascular and macrovascular complications often occur concomitantly, and share similar risk factors and pathological pathways [3-5]. The presence of microvascular disease increases the risk of cardiovascular morbidity and mortality in people with type 2 diabetes, independent of the major established cardiovascular risk factors [6]. However, the impact of macrovascular disease on the risk of microvascular events has not been fully investigated. We sought to evaluate the impact of microvascular and macrovascular disease, considered individually, and together, on the risk of death and major macrovascular and microvascular events in patients with type 2 diabetes participating in the Action in Diabetes and Vascular Disease: PreterAx and DiamicroN Modified-Release Controlled Evaluation (ADVANCE) trial (ClinicalTrials.gov Number, NCT00145925) and ADVANCE-ON post-trial study (ClinicalTrials.gov Number, NCT00949286).

\section{Materials and methods Participants}

ADVANCE was a multi-national randomized trial testing the effect of intensive glucose control (using a gliclazide$\mathrm{MR}$ ) and routine blood pressure treatment (using a fixeddose combination of perindopril and indapamide) on the risk of major microvascular and macrovascular events in 11,140 patients with type 2 diabetes and at least one other cardiovascular risk factor or pre-existing cardiovascular disease [7-9]. Subsequently, 8494 of the surviving participants were enrolled in the post-trial observational study (ADVANCE-ON). The design and characteristics of participants have been previously described [8-10]. The Institutional Ethics Committee of each participating centre approved the ADVANCE and ADVANCE-ON protocols, and all participants provided written informed consent.

\section{Definition of microvascular and macrovascular disease at baseline}

At baseline, microvascular disease was defined as the presence of macroalbuminuria (urinary albumin to creatinine ratio $(\mathrm{ACR})>300 \mathrm{mg} / \mathrm{g}$ ), requirement of retinal photocoagulation therapy, proliferative retinopathy, macular oedema, or diabetes-related blindness. Macrovascular disease was defined as the presence, at baseline, of myocardial infarction, stroke, coronary artery bypass graft, percutaneous transluminal coronary angioplasty, hospital admission for unstable angina or transient ischaemic attack, lower-extremity amputation of at least one digit secondary to arterial insufficiency, or a peripheral revascularisation procedure. Participants were categorized into four baseline groups: absence of both microvascular and macrovascular disease, presence of microvascular disease alone, presence of macrovascular disease alone, and presence of both microvascular and macrovascular disease.

\section{Definition of outcomes}

The primary outcomes were all-cause mortality, major macrovascular events (MACE: a composite of nonfatal myocardial infarction, nonfatal stroke, or cardiovascular death), and major clinical microvascular events (a composite of end-stage renal disease (ESRD), defined as requirement for renal-replacement therapy; death induced by renal disease; requirement for retinal photocoagulation; or diabetes-related blindness in either eye). The secondary outcomes were cardiovascular death, fatal or nonfatal myocardial infarction, fatal or nonfatal stroke, ESRD or renal death, and requirement for retinal photocoagulation or blindness. Outcomes were adjudicated by an independent End Point Adjudication Committee in the ADVANCE trial, through to the end of randomized allocation, and were reported by investigators without adjudication in the ADVANCE-ON study, in accordance with its pre-specified protocol [10].

\section{Statistical analyses}

Continuous variables were summarized as mean (SD) or, for those with a skewed distribution, median (interquartile range). Categorical variables were summarized as the number of patients with corresponding percentage. Characteristics of participants according to status of microvascular and macrovascular disease at baseline were compared using Chi squared, ANOVA, or KruskalWallis tests.

Cumulative incidence curves were used to plot survival (outcome-free) rates during follow-up, and compared using the log-rank test. Cox proportional hazards regression models were fitted to estimate hazard ratios (HRs), with associated 95\% confidence intervals (CI), for outcomes by joint status of microvascular and macrovascular disease at baseline. Analyses were adjusted for randomized study allocations plus every potential confounding variable that was significantly different between microvascular and macrovascular disease at baseline: sex, age, region of origin (Asia: Philippines, China, Malaysia, and India; established market economies: Australia, Canada, France, Germany, Ireland, Italy, Netherlands, New Zealand, United Kingdom; and Eastern Europe: Czech Republic, Estonia, Hungary, Lithuania, Poland, Russia, Slovakia), body mass index, duration of diabetes, HbA1c, 
systolic blood pressure, antihypertensive treatment, estimated glomerular filtration rate (eGFR; computed by the Chronic Kidney Disease-Epidemiology Collaboration equation [11]) and its square, urinary ACR, LDL- and HDL-cholesterol, and history of ever smoking (basic model). The proportional hazards assumption was checked using the Schoenfeld residuals method.

We tested multiplicative interaction between baseline history of microvascular disease and macrovascular disease on the risk of each outcome by including these two individual variables and their product within Cox models.

Harrell's c-statistic [12], net reclassification improvement (NRI) and integrated discrimination improvement (IDI) were used to compare discrimination and classification of primary outcomes, assessed using survival methodology, between two prognostic models: basic model and basic model plus baseline history of microvascular or macrovascular disease (as appropriate). Since we assessed the additive value of microvascular disease (including macroalbuminuria) on the risk of all-cause mortality and MACE, urinary ACR was not included in the basic model in these analyses. We also evaluated the individual and joint prognostic value of urinary ACR (as a continuous variable) and diabetic retinopathy, added to the basic model, on the discrimination of all-cause mortality and MACE.

Sensitivity analyses were conducted after including further components of microvascular disease at baseline: (i) chronic kidney disease (CKD defined as eGFR $<60 \mathrm{ml} /$ $\mathrm{min} / 1.73 \mathrm{~m}^{2}$ ) or (ii) diabetic peripheral neuropathy (defined as a disturbance of 10-g monofilament sensation or absence of ankle reflex in both feet). We also evaluated the associations of microvascular and macrovascular disease at baseline on the risk of MACE and major clinical microvascular events after treating non-renal and noncardiovascular death as a competing risk using the Fine and Gray method [13].

Statistical analyses were performed using SAS software, version 9.3 (SAS Institute, www.sas.com), and Stata software version 13 (StataCorp, www.stata.com). A P value less than 0.05 was considered significant.

\section{Results}

\section{Clinical characteristics at baseline}

Among 11,140 participants enrolled in ADVANCE trial, 761 (6.8\%) had microvascular disease alone, 3196 (28.7\%) had macrovascular disease alone, and 394 (3. 5\%) had both at baseline (Additional file 1: Table S1). Patients with microvascular disease alone at baseline had a longer duration of diabetes and higher HbA1c, systolic blood pressure, and ACR levels, whereas those with macrovascular disease alone were more frequently men, from established market economies, ever smokers, and treated with antihypertensive, lipid lowering and antiplatelet drugs (Table 1).

\section{Incidence of major outcomes during follow-up according to status of microvascular and macrovascular disease at baseline}

All-cause mortality, MACE, and major clinical microvascular events occurred in 2265 (20.3\%), 2166 (19.4\%), and 807 (7.2\%) participants, respectively, during a median overall follow-up of 9.9 (inter-quartile interval $5.6-10.9)$ years. Their incidence rates were $2.4,2.4$, and 0.9 per 100 person-years, respectively. The risks of these respective outcomes were higher in patients with baseline history of only microvascular (HRs [95\% CI] 1.43 [1.201.71 ], $\mathrm{p}<0.0001 ; 1.64$ [1.37-1.97], $\mathrm{p}<0.0001$; and 4.74 $[3.86-5.82],<0.0001)$ and of only macrovascular disease (1.43 [1.30-1.57], $\mathrm{p}<0.0001 ; 2.04$ [1.86-2.25], $\mathrm{p}<0.0001$; and $1.26[1.06-1.51], \mathrm{p}=0.01)$, compared with patients without either of these conditions (Fig. 1; Table 2). The highest risks were observed in patients with both conditions at baseline $(2.01$ [1.65-2.45], $\mathrm{p}<0.0001 ; 2.92$ [2.403.55], $\mathrm{p}<0.0001$; and 6.30 [4.93-8.06], $\mathrm{p}<0.0001$ ). There was no evidence of interaction between microvascular and macrovascular disease at baseline on the risks of allcause mortality $(p=0.89)$, MACE $(p=0.25)$, or major clinical microvascular events $(\mathrm{p}=0.75)$.

Comparable results were observed with secondary endpoints, except for the absence of association of baseline macrovascular disease with the risk of ESRD or renal death (Table 2).

\section{Additive values of microvascular or macrovascular disease at baseline in discrimination and classification of outcomes during follow-up}

The addition of microvascular disease (change in c-statistic [95\% CI] $0.005[0.002-0.008], \mathrm{p}=0.02)$ or macrovascular disease $(0.005$ [0.002-0.007], $\mathrm{p}<0.0001)$ considered separately or together $(0.011$ [0.007-0.014], $\mathrm{p}<0.0001)$ improved the discrimination, as well as the classification (IDI: 0.013 [0.010-0.016], p < 0.001; NRI: 0.021 [0.011-0.032], $\mathrm{p}<0.001)$ of the risk of all-cause mortality. The addition of ACR (0.010 [0.006-0.014], $\mathrm{p}<0.0001)$ displayed a higher improvement of death discrimination than diabetic retinopathy $(0.002$ [0.0010.004], $\mathrm{p}=0.01$ ).

The addition of baseline microvascular disease to established cardiovascular risk factors improved the discrimination (0.009 [0.003-0.014], $\mathrm{p}=0.002)$ and classification (IDI: 0.008 [0.006-0.010], p < 0.001; NRI: 0.011 [0.001-0.020], $\mathrm{p}=0.02)$ of MACE. The improvement in discrimination of MACE was similar after addition 
Table 1 Clinical characteristics according to microvascular or macrovascular disease at baseline

\begin{tabular}{|c|c|c|c|c|c|c|}
\hline & Overall & History of micr & ascular or macrov & scular disease at b & line & \\
\hline & $11,140)$ & $\begin{array}{l}\text { Dual absence } \\
(n=6789)\end{array}$ & $\begin{array}{l}\text { Microvascular } \\
\text { alone }(n=761)\end{array}$ & $\begin{array}{l}\text { Macrovascular } \\
\text { alone }(n=3196)\end{array}$ & $\begin{array}{l}\text { Both } \\
(n=394)\end{array}$ & $\mathrm{p}$ \\
\hline Male sex, n (\%) & $6407(57.5)$ & $3628(53.4)$ & $404(53.1)$ & $2107(65.9)$ & $268(68.0)$ & $<0.0001$ \\
\hline Region of origin: Asia, $n$ (\%) & $4136(37.1)$ & $2525(37.2)$ & $350(46.0)$ & $1115(34.9)$ & $146(37.1)$ & $<0.0001$ \\
\hline $\begin{array}{l}\text { Region of origin: established market } \\
\text { economies, } \mathrm{n}(\%)\end{array}$ & $4862(43.7)$ & $3012(44.4)$ & $292(38.4)$ & $1389(43.5)$ & $169(42.9)$ & \\
\hline Region of origin: Eastern Europe, n (\%) & $2142(19.2)$ & $1252(18.4)$ & $119(15.6)$ & $692(21.6)$ & $79(20.0)$ & \\
\hline Age (years): mean (SD) & $65.8(6.4)$ & $65.9(6.3)$ & $65.3(6.4)$ & $65.6(6.6)$ & $67.0(6.6)$ & $<0.0001$ \\
\hline Duration of diabetes (years): mean (SD) & $7.9(6.4)$ & $7.6(6.1)$ & $10.3(7.3)$ & $7.7(6.3)$ & $10.2(7.2)$ & $<0.0001$ \\
\hline Body mass index $\left(\mathrm{kg} / \mathrm{m}^{2}\right)$ : mean (SD) & $28.3(5.2)$ & $28.4(5.3)$ & $27.7(5.2)$ & $28.4(4.9)$ & $28.2(5.4)$ & 0.002 \\
\hline $\begin{array}{l}\text { Systolic blood pressure }(\mathrm{mmHg}) \text { : mean } \\
\text { (SD) }\end{array}$ & $145(22)$ & $145(21)$ & $149(24)$ & $144(22)$ & $148(23)$ & $<0.0001$ \\
\hline $\begin{array}{l}\text { Diastolic blood pressure }(\mathrm{mmHg}) \text { : mean } \\
\text { (SD) }\end{array}$ & $81(11)$ & $81(11)$ & $81(12)$ & $80(11)$ & $81(11)$ & 0.41 \\
\hline Use of antihypertensive treatment, n (\%) & $7655(68.7)$ & $4357(64.2)$ & $521(68.5)$ & $2466(77.2)$ & $311(78.9)$ & $<0.0001$ \\
\hline HbA1c (\%): mean (SD) & $7.5(1.6)$ & $7.5(1.6)$ & $7.9(1.7)$ & $7.4(1.5)$ & $7.9(1.6)$ & $<0.0001$ \\
\hline HbA1c (mmol/mol): mean (SD) & $59(17)$ & $58(17)$ & $63(19)$ & $57(16)$ & $62(18)$ & \\
\hline $\mathrm{eGFR}\left(\mathrm{ml} / \mathrm{min} / 1.73 \mathrm{~m}^{2}\right)$ & $74(18)$ & $76(17)$ & $73(20)$ & $73(18)$ & $69(20)$ & $<0.0001$ \\
\hline Urinary ACR (mg/g): median (Q1, Q3) & $15(7,40)$ & $13(7,31)$ & $49(12,390)$ & $15(7,36)$ & $86(13,461)$ & $<0.0001$ \\
\hline $\begin{array}{l}\text { Serum total cholesterol (mmol/l): mean } \\
\text { (SD) }\end{array}$ & $5.2(1.2)$ & $5.3(1.2)$ & $5.3(1.2)$ & $5.0(1.2)$ & $4.9(1.2)$ & $<0.0001$ \\
\hline $\begin{array}{l}\text { Serum HDL cholesterol (mmol/l): mean } \\
\text { (SD) }\end{array}$ & $1.3(0.4)$ & $1.3(0.4)$ & $1.3(0.4)$ & $1.2(0.3)$ & $1.2(0.3)$ & $<0.0001$ \\
\hline $\begin{array}{l}\text { Serum LDL cholesterol (mmol/l): mean } \\
\text { (SD) }\end{array}$ & $3.1(1.0)$ & $3.2(1.0)$ & $3.2(1.0)$ & $3.0(1.1)$ & $2.9(1.0)$ & $<0.0001$ \\
\hline $\begin{array}{l}\text { Serum triglycerides (mmol/l): median } \\
(\mathrm{Q} 1, \mathrm{Q} 3)\end{array}$ & $1.6(1.2,2.3)$ & $1.6(1.2,2.3)$ & $1.6(1.2,2.3)$ & $1.7(1.2,2.3)$ & $1.6(1.1,2.2)$ & 0.05 \\
\hline History of ever smoking, n (\%) & $4674(42.0)$ & $2702(39.8)$ & $271(35.6)$ & $1529(47.8)$ & $172(43.7)$ & $<0.0001$ \\
\hline
\end{tabular}

Comparison of qualitative and quantitative parameters were performed using Chi square and ANOVA tests, respectively. Kruskal-Wallis test was used for variables with skewed distribution (urinary albumin-creatinine ratio and triglycerides). $p<0.05$ was significant

Established market economies: Australia, Canada, France, Germany, Ireland, Italy, Netherlands, New Zealand, United Kingdom; Eastern Europe: the Czech Republic, Estonia, Hungary, Lithuania, Poland, Russia, Slovakia; Asia: Philippines, China, Malaysia, India. eGFR, computed by the Chronic Kidney Disease Epidemiology Collaboration equation. ACR, Albumin to Creatinine Ratio. Use of lipid lowering drugs: statins or other hypolipidemic agents

of either urinary ACR (0.008 [0.003-0.013], $\mathrm{p}=0.002)$ or diabetic retinopathy $(0.007$ [0.002-0.011], $\mathrm{p}=0.005)$. Adding both urinary ACR and diabetic retinopathy together yielded the highest increase in discrimination of MACE (0.014 [0.007-0.021], $\mathrm{p}<0.0001)$.

Baseline macrovascular disease modestly enhanced IDI (0.002 [0.001-0.002]), $\mathrm{p}<0.001$ and NRI (0.041 [0.0020.087], $\mathrm{p}<0.0001$ ), but not discrimination (c-statistic), of major clinical microvascular events (Table 3).

\section{Sensitivity analyses}

Comparable associations were observed between baseline microvascular disease, including CKD or peripheral diabetic neuropathy, and the risk of outcomes, except for myocardial infarction or stroke (Additional file 1: Tables S2, S3). Baseline microvascular or macrovascular disease remained significantly associated with MACE and major clinical microvascular events when we corrected for competing risk of non-renal and non-cardiovascular death (Additional file 1: Table S4).

\section{Discussion}

This study demonstrates the independent association of microvascular or macrovascular disease at baseline with excess risks of all-cause mortality, MACE, and major clinical microvascular events in patients with type 2 diabetes followed for a median duration of 9.9 years. The presence of both conditions led to the highest risks. Baseline microvascular disease enhanced discrimination and classification of MACE, while baseline macrovascular disease modestly improved classification, but not discrimination, of major clinical microvascular events. Overall, the improvement in discrimination and classification of outcomes was quiet modest, but statistically significant. Interestingly, urinary ACR and diabetic retinopathy yielded together the highest improvement of 

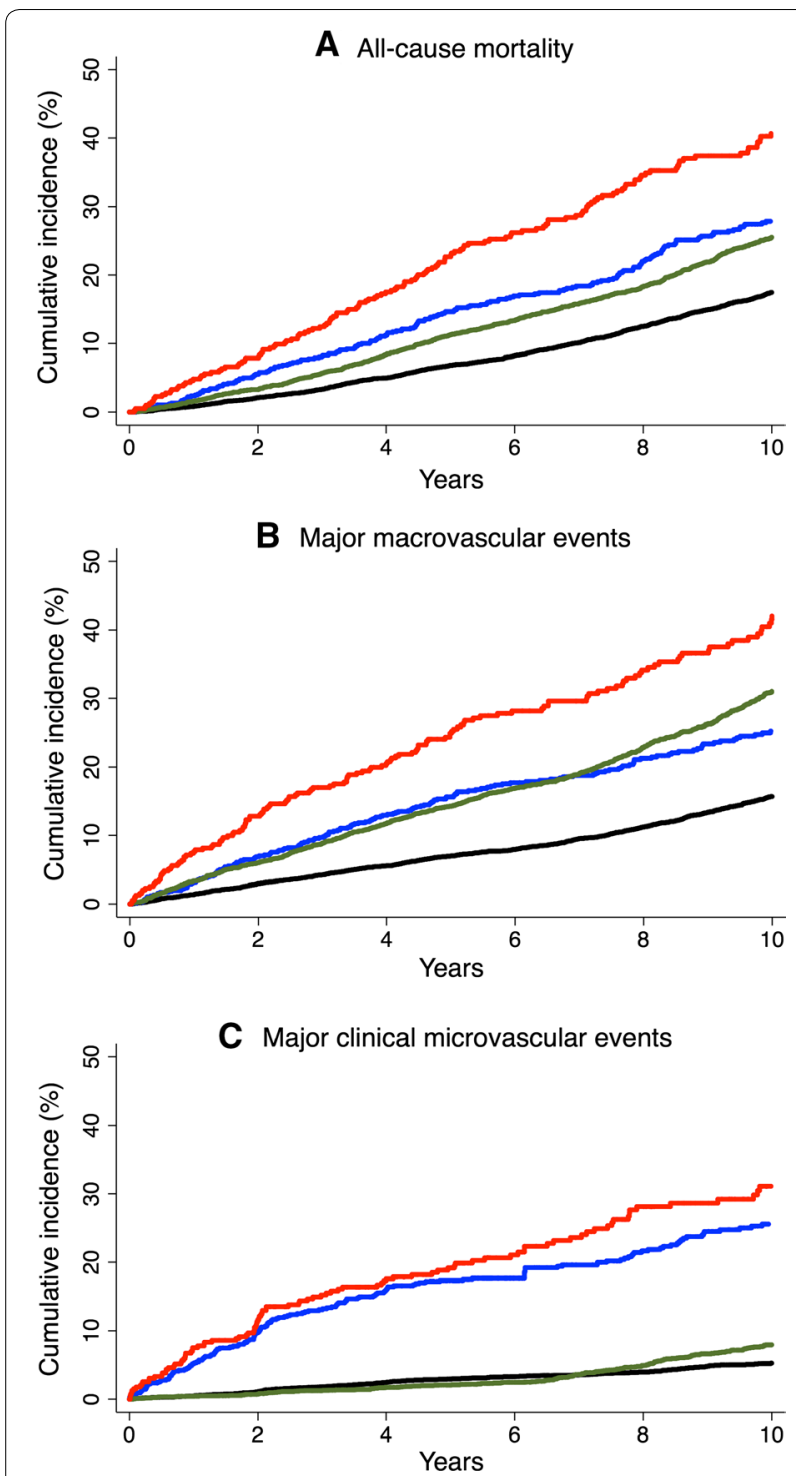

Fig. 1 Cumulative incidence of outcomes during follow-up according to status of microvascular and macrovascular disease at baseline. a All-cause mortality. b Major macrovascular events. c Major clinical microvascular disease ( $p<0.0001$ for all). Black line absence of both macrovascular and microvascular disease. Blue line presence of microvascular disease alone. Green line presence of macrovascular disease alone. Red line presence of both microvascular and macrovascular disease

MACE supporting their additive value in the prediction of macrovascular disease. Of note, both ACR and retinopathy were independent components of the 10-year prognostic vascular risk score recently reported by our group in the ADVANCE-ON stud [14].

\section{Microvascular disease and risk of MACE}

Our findings support previous studies showing that microvascular disease is an important predictor of future macrovascular disease and death $[6,15]$. ADVANCE participants with microvascular disease alone at baseline displayed similar hazard ratios for all-cause mortality or MACE, compared to those with macrovascular disease alone, suggesting that microvascular impairment plays an important role in the development of diabetic angiopathy. A recent population-based cohort study has shown that retinal and skin microvascular abnormalities occurred early in prediabetes, were more severe in type 2 diabetes, and may contribute to the development of cardiovascular disease [16]. Other studies reported a high prevalence of coronary microvascular dysfunction in patients with type 2 diabetes free for known cardiovascular disease [17, 18].

\section{Macrovascular disease and risk of major clinical microvascular events}

We evaluated, for the first time, the impact of macrovascular disease at baseline on the 9.9-year risk of major clinical microvascular events in patients with type 2 diabetes. Macrovascular disease at baseline was significantly associated with an elevated risk of the composite major microvascular endpoint, as well as retinal photocoagulation or blindness, but not ESRD or renal death. We recently reported that the history of peripheral arterial disease was associated with the risk of severe diabetic retinopathy, but not renal endpoints in ADVANCE and ADVANCE-ON studies [19]. Based on their poor prognosis, patients with chronic macrovascular disease at baseline may have died before experiencing ESRD during the follow-up. The impact of macrovasular disease on the risk of major clinical microvascular events seems to be weaker than the effect of microvascular disease on MACE risk, but there was no evidence of an interaction between the two conditions at baseline on the risk of any measured outcome. Despite their common background, these conditions had independent effects, suggesting that different mechanisms may be involved in the impact of microvascular and macrovascular disease on future vascular events. Hence there is a need to evaluate new predictors for both microvascular and macrovascular events. For instance, a high index of microcirculatory resistance and low coronary flow reserve were recently found associated with poor cardiovascular prognosis in patients with intermediate coronary stenosis (29\% with type 2 diabetes) [20].

\section{Physiopathological mechanisms linking microvascular and macrovascular disease}

Several pathways may explain the relationship between microvascular and macrovascular disease in patients with diabetes. Diabetic microvascular complications are mainly caused by prolonged exposure to high glucose levels. Diabetes is also associated with 


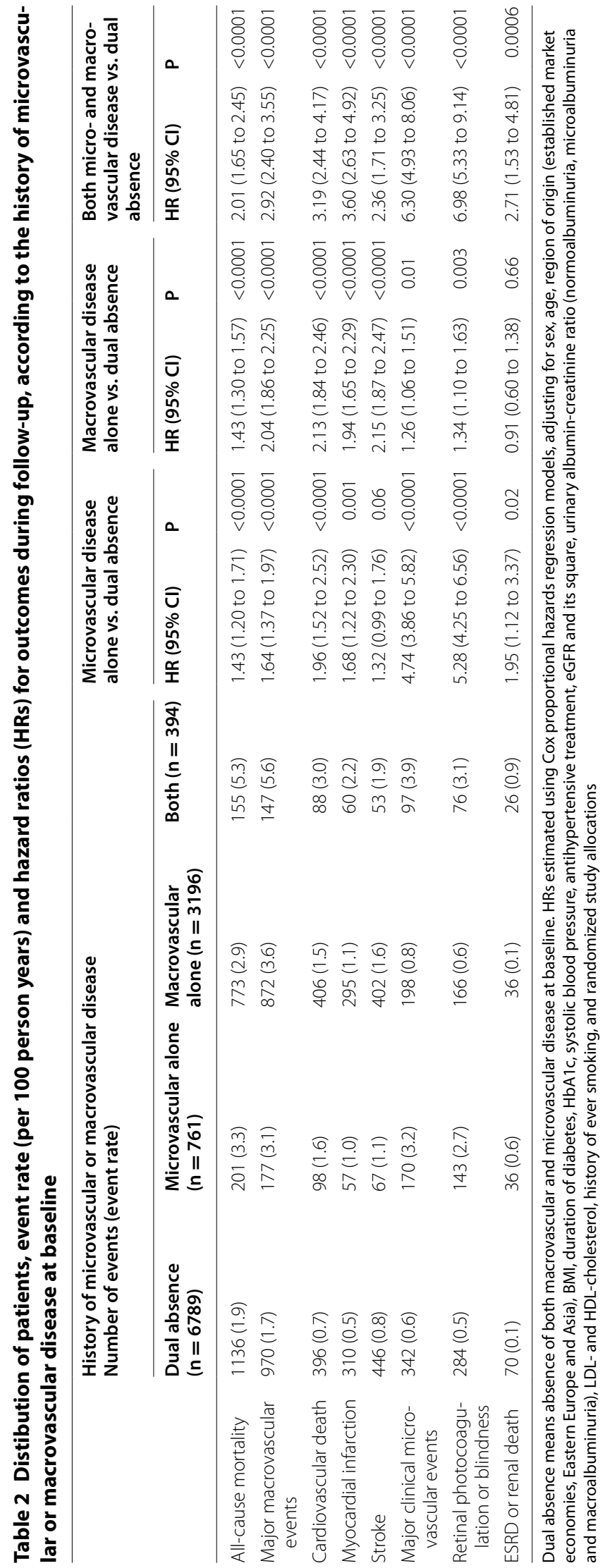


Table 3 Harrell's c-statistics, NRI, IDI for risk of major outcomes according to traditional risk factors without and with history of microvascular or macrovascular disease at baseline

\begin{tabular}{|c|c|c|}
\hline \multicolumn{2}{|l|}{ Risk of all-cause mortality } & \multirow[t]{2}{*}{$\mathbf{P}$} \\
\hline C-statistic $(95 \%$ Cl) for basic model & 0.704 (0.693 to 0.716$)$ & \\
\hline Change in C-statistic ( $95 \% \mathrm{Cl}$ ) for basic model + microvascular disease & 0.005 (0.002 to 0.008) & 0.02 \\
\hline Change in C-statistic (95\% Cl) for basic model + macrovascular disease & 0.005 (0.002 to 0.007$)$ & $<0.0001$ \\
\hline Change in C-statistic $(95 \% \mathrm{Cl}$ ) for basic model + microvascular disease + macrovascular disease & 0.011 (0.007 to 0.014$)$ & $<0.0001$ \\
\hline IDI $(95 \% \mathrm{Cl})$ & 0.013 (0.010 to 0.016$)$ & $<0.001$ \\
\hline Continuous NRI (95\% Cl) & 0.275 (0.227 to 0.325$)$ & $<0.001$ \\
\hline Categorical NRI (95\% Cl) & 0.021 (0.011 to 0.032) & $<0.001$ \\
\hline \multicolumn{3}{|l|}{ Risk of major macrovascular events (MACE) } \\
\hline C-statistic ( $95 \% \mathrm{Cl})$ for basic model & 0.648 (0.631 to 0.665$)$ & \\
\hline Change in C-statistic ( $95 \% \mathrm{Cl}$ ) for basic model + microvascular disease & 0.009 (0.003 to 0.014$)$ & 0.002 \\
\hline IDI (95\% Cl) & 0.008 (0.006 to 0.010$)$ & $<0.001$ \\
\hline Continuous NRI (95\% Cl) & 0.120 (0.073 to 0.167$)$ & $<0.001$ \\
\hline Categorical NRI $(95 \% \mathrm{Cl})$ & 0.011 (0.001 to 0.020$)$ & 0.02 \\
\hline \multicolumn{3}{|l|}{ Risk of major clinical microvascular events } \\
\hline C-statistic $(95 \% \mathrm{Cl})$ for basic model & 0.664 (0.639 to 0.689$)$ & \\
\hline Change in C-statistic ( $95 \%$ Cl) for basic model + macrovascular disease & $0.004(-0.003$ to 0.011$)$ & 0.25 \\
\hline IDI $(95 \% \mathrm{Cl})$ & 0.002 (0.001 to 0.002$)$ & $<0.001$ \\
\hline Continuous NRI (95\% Cl) & 0.211 (0.112 to 0.305$)$ & $<0.001$ \\
\hline Categorical NRI (95\% Cl) & 0.041 (0.002 to 0.087$)$ & 0.03 \\
\hline
\end{tabular}

accelerated atherosclerosis affecting large vessels [21, 22]. Atherosclerosis is more prevalent in diabetic patients with microvascular disease compared to those without $[21,22]$. However, it is still unknown why intensive glucose control does not yield the same benefit on macrovascular events as observed for microvascular outcomes [23]. Chronic hyperglycaemia causes vascular damage through activation of major biochemical paths including polyol pathway flux, increased formation of advanced glycation end products (AGEs), increased expression of AGEs receptor and its activating ligands, activation of protein kinase $\mathrm{C}$ isoforms, and overactivity of the hexosamine pathway [24]. Numerous lines of evidence suggest that these biochemical abnormalities may be activated by mitochondrial overproduction of reactive oxygen species (ROS) induced by hyperglycaemia [25]. The excess of ROS production with decreased in antioxidant capacity lead to oxidative stress, which plays an important role in the premature vascular morbidity and mortality in patients with diabetes [26]. Oxidative stress impairs endothelial function and endothelium-dependent vasodilation by inactivation of $\mathrm{NO}$, and induces cell proliferation, hypertrophy, cardiac remodeling, apoptosis, and low-grade inflammation in endothelial and smooth cells of the vascular wall [27-30]. Moreover, oxidative stress is associated with many other accelerating conditions including insulin resistance, metabolic syndrome, hypertension, dyslipidaemia, and obesity, leading to both microvascular and macrovascular disease [25, 31]. The depletion of circulating stem cells may also explain the association between microvascular and macrovascular disease. A recent study has shown lower CD34 ${ }^{+}$and $\mathrm{CD}_{34}{ }^{+} \mathrm{CD} 133^{+}$cells in type 2 diabetic patients with cardiovascular events compared to those without [32]. The reduced levels of circulating progenitor cells improve the prediction of both macrovascular and microvascular events [32, 33]. The remodeling of bone marrow involves neurovascular changes and vascular abnormalities comparable to the known microangiopathy seen in the kidney and the retina [34].

\section{Strengths and limitations}

The main strength of our study is the evaluation of the individual and the combined impact of microvascular and macrovascular disease at baseline on the risk of death and major microvascular and macrovascular outcomes in a large international cohort of patients with type 2 diabetes followed for a median duration of 9.9 years. The main 
limitation is the absence of biochemical renal assessment during the ADVANCE-ON follow-up, which may underestimate the association between baseline macrovascular disease and kidney disease. Furthermore, peripheral diabetic neuropathy was not investigated as an outcome in the post-trial, ADVANCE-ON, observational study. Although comparable findings were observed when we evaluated the associations of microvascular disease at baseline, including the history of peripheral diabetic neuropathy, with the main outcomes.

\section{Conclusion}

The presence of microvascular or macrovascular disease at baseline is independently associated with increased risk of death, MACE, and major clinical microvascular events in people with type 2 diabetes followed for a median duration of 9.9 years, and their coexistence has an additive prognostic value. These findings encourage consideration of prior microvascular disease including retinal status, in addition to traditional cardiovascular risk factors, in selecting participants for forthcoming clinical trials aiming to assess vascular endpoints in patients with type 2 diabetes.

\section{Additional file}

Additional file 1: Table S1. Components of microvascular and macrovascular disease at baseline. Table S2. Distibution of patients, event rate (per 100 person years) and hazard ratios (HRs) for outcomes during follow-up, according to the history of microvascular (including chronic kidney disease) or macrovascular disease at baseline. Table S3. Distibution of patients, event rate (per 100 person years) and hazard ratios (HRs) for outcomes during follow-up, according to the history of microvascular (including peripheral neuropathy) or macrovascular disease at baseline. Table S4. Subdistribution hazard ratios for outcomes during follow-up according to the history of microvascular or macrovascular disease at baseline with correction for competing risk of non-renal and non-cardiovascular death.

\section{Abbreviations}

AGEs: advanced glycation end products; ACR: albumin to creatinine ratio; ADVANCE: Action in Diabetes and Vascular Disease: PreterAx and DiamicroN Modified-Release Controlled Evaluation trial; CKD: chronic kidney disease; $\mathrm{Cl}$ : confidence interval; eGFR: estimated glomerular filtration rate; ESRD: end stage renal disease; HR: hazard ratio; IDI: integrated discrimination improvement; MACE: major macrovascular events; NRI: net reclassification improvement; ROS: reactive oxygen species; SD: standard deviation.

\section{Authors' contributions}

$\mathrm{KM}$ wrote the manuscript with assistance from $\mathrm{MW}$ and $\mathrm{JC} ; \mathrm{KM}$ and JC designed the study; MM, SC, MC, SH, GM, NP, BW, and SZ contributed to the discussion and reviewed the manuscript. JC and $\mathrm{KM}$ are the guarantors of this work and, as such, had full access to all the data in the study and take responsibility for the integrity of the data and the accuracy of the data analysis. All authors read and approved the final manuscript.

\section{Author details}

${ }^{1}$ The George Institute for Global Health, University of Sydney, Sydney, NSW, Australia. ${ }^{2}$ INSERM, UMRS 1138, Centre de Recherche des Cordeliers, Paris,
France. ${ }^{3}$ Department of Diabetology, Endocrinology and Nutrition, Assistance Publique Hôpitaux de Paris, Bichat Hospital, DHU FIRE, Paris, France. ${ }^{4}$ The George Institute for Global Health, University of Oxford, Oxford, UK. ${ }^{5}$ Department of Epidemiology, Johns Hopkins Bloomberg School of Public Health, Johns Hopkins University, Baltimore, MD, USA. ${ }^{6}$ University Paris Diderot, Sorbonne Paris Cité, UFR de Médecine, Paris, France. ${ }^{7}$ Boden Institute of Obesity, Nutrition, Exercise \& Eating Disorders, Sydney Medical School, University of Sydney, Sydney, NSW, Australia. ${ }^{8}$ Baker IDI Heart and Diabetes Institute, Melbourne, VIC, Australia. ${ }^{9}$ The University of Melbourne and Royal Melbourne Hospital, Melbourne, VIC, Australia. ${ }^{10}$ The University of Milan-Bicocca and Istituto Auxologico Italiano, Milan, Italy. ${ }^{11}$ The International Centre for Circulatory Health, National Heart and Lung Institute, Imperial College, London, UK. ${ }^{12}$ Institute of Cardiovascular Sciences, University College London (UCL) and NIHR UCL Hospitals Biomedical Research Centre, London, UK. ${ }^{13}$ Monash Centre for Health Research and Implementation, School of Public Health and Preventive Medicine, Monash University, Clayton, VIC, Australia.

\section{Acknowledgements}

KM was supported by grants from the Société Francophone du Diabète (SFD) and the Association Diabète Risque Vasculaire (ADRV), and travel support from the Association pour le Développement de l'Enseignement et des Recherches auprès des universités, des centres de recherches et des entreprises d'Aquitaine (ADERA).

\section{Competing interests}

Dr. Kamel Mohammedi reports personal fees from Novo-Nordisk, outside the submitted work; Prof. Marc Woodward reports personal fees from Amgen, outside the submitted work; Prof. Michel Marre reports grants and personal fees from Novo Nordisk, grants and personal fees from Sanofi, grants and personal fees from Eli Lilly, personal fees from Servier, grants and personal fees from Merck Sharp and Dohme, personal fees from Abbott, grants and personal fees from Novartis, personal fees from Astra Zeneca, outside the submitted work; Prof. Stephen Harrap reports grants from National Health and Medical Research Council of Australia, grants from The George Institute for Global Health, during the conduct of the study; other from Servier, outside the submitted work; Prof. Neil Poulter reports grants from The George Institute, grants from British Heart Foundation/Diabetes UK/The George Institute, during the conduct of the study; grants from The George Institute, grants from BHF/ DUK/The George Institute, outside the submitted work; Prof. Bryan Williams reports lecture fees from Servier, Novartis, Beoringher Ingelheim and Diachi Sankyo, outside the submitted work; and Prof. John Chalmers reports grants from National Health and Medical Research Council of Australia, grants and personal fees from Servier, outside the submitted work. No other potential conflict of interest relevant to this article was reported.

\section{Availability of data and materials}

The datasets analysed during the current study are not publicly available due to consideration of intellectual property, due to many ongoing active collaborations worldwide, and to continuing analyses by the study investigators, but may be available from the principal investigator on reasonable request.

\section{Consent for publication}

Not applicable.

\section{Ethics approval and consent to participate}

The Institutional Ethics Committee of each participating centre approved the ADVANCE and ADVANCE-ON protocols, and all participants provided written informed consent.

\section{Funding}

Not applicable.

\section{Publisher's Note}

Springer Nature remains neutral with regard to jurisdictional claims in published maps and institutional affiliations.

Received: 8 May 2017 Accepted: 21 July 2017

Published online: 27 July 2017 


\section{References}

1. Cordero A, Lopez-Palop R, Carrillo P, Moreno-Arribas J, Bertomeu-Gonzalez V, Frutos A, Garcia-Carrilero M, Gunturiz C, Bertomeu-Martinez V. Comparison of long-term mortality for cardiac diseases in patients with versus without diabetes mellitus. Am J Cardiol. 2016;117(7):1088-94.

2. Bots SH, van der Graaf Y, Nathoe HM, de Borst GJ, Kappelle JL, Visseren FL, Westerink J. The influence of baseline risk on the relation between $\mathrm{HbA} 1 \mathrm{c}$ and risk for new cardiovascular events and mortality in patients with type 2 diabetes and symptomatic cardiovascular disease. Cardiovasc Diabetol. 2016;15(1I):101

3. Krentz AJ, Clough G, Byrne CD. Interactions between microvascular and macrovascular disease in diabetes: pathophysiology and therapeutic implications. Diabetes Obes Metab. 2007;9(61):781-91.

4. Bramlage P, Gitt AK, Schneider S, Deeg E, Tschope D. Clinical course and outcomes of type-2 diabetic patients after treatment intensification for insufficient glycaemic control_-results of the 2 year prospective DiaRegis follow-up. BMC Cardiovasc Disord. 2014;14:162.

5. Kontopantelis E, Springate DA, Reeves D, Ashcroft DM, Rutter MK, Buchan I, Doran T. Glucose, blood pressure and cholesterol levels and their relationships to clinical outcomes in type 2 diabetes: a retrospective cohort study. Diabetologia. 2015;58(31):505-18.

6. Brownrigg JR, Hughes CO, Burleigh D, Karthikesalingam A, Patterson BO, Holt PJ, Thompson MM, de Lusignan S, Ray KK, Hinchliffe RJ. Microvascular disease and risk of cardiovascular events among individuals with type 2 diabetes: a population-level cohort study. Lancet Diabetes Endocrinol. 2016;4(7l):588-97.

7. ADVANCE Management Committee. Study rationale and design of ADVANCE: action in diabetes and vascular disease-preterax and diamicron MR controlled evaluation. Diabetologia. 2001;44(91):1118-20.

8. Patel A, MacMahon S, Chalmers J, Neal B, Billot L, Woodward M, Marre M, Cooper M, Glasziou P, Grobbee D, et al. Intensive blood glucose control and vascular outcomes in patients with type 2 diabetes. N Engl J Med. 2008:358(241):2560-72.

9. Patel A, MacMahon S, Chalmers J, Neal B, Woodward M, Billot L, Harrap S, Poulter N, Marre M, Cooper M, et al. Effects of a fixed combination of perindopril and indapamide on macrovascular and microvascular outcomes in patients with type 2 diabetes mellitus (the ADVANCE trial): a randomised controlled trial. Lancet. 2007;370(9590I):829-40.

10. Zoungas S, Chalmers J, Neal B, Billot L, Li Q, Hirakawa Y, Arima H, Monaghan $\mathrm{H}$, Joshi R, Colagiuri $\mathrm{S}$, et al. Follow-up of blood-pressure lowering and glucose control in type 2 diabetes. N Engl J Med. 2014:371(15I):1392-406

11. KDIGO Group. KDIGO 2012 clinical practice guideline for the evaluation and management of chronic kidney disease. Kidney Inter. 2013;3(1):1-150

12. Pencina MJ, D'Agostino RB. Overall $C$ as a measure of discrimination in survival analysis: model specific population value and confidence interval estimation. Stat Med. 2004;23(131):2109-23.

13. Fine JP, Gray RJ. A proportional hazards model for the subdistribution of a competing risk. J Am Stat Assoc. 1999;94:496-509.

14. Woodward M, Hirakawa Y, Kengne AP, Matthews DR, Zoungas S, Patel A Poulter N, Grobbee R, Cooper M, Jardine M, et al. Prediction of 10-year vascular risk in patients with diabetes: the AD-ON risk score. Diabetes Obes Metab. 2016;18(31):289-94

15. Mohammedi K, Woodward M, Hirakawa Y, Zoungas S, Williams B, Lisheng L, Rodgers A, Mancia G, Neal B, Harrap S, et al. Microvascular and macrovascular disease and risk for major peripheral arterial disease in patients with type 2 diabetes. Diabetes Care. 2016;39(10l):1796-803.

16. Sorensen BM, Houben AJ, Berendschot TT, Schouten JS, Kroon AA, van der Kallen CJ, Henry RM, Koster A, Sep SJ, Dagnelie PC, et al. Prediabetes and type 2 diabetes are associated with generalized microvascular dysfunction: the maastricht study. Circulation. 2016;134(181):1339-52.

17. von Scholten BJ, Hasbak P, Christensen TE, Ghotbi AA, Kjaer A, Rossing P, Hansen TW. Cardiac (82)Rb PET/CT for fast and non-invasive assessment of microvascular function and structure in asymptomatic patients with type 2 diabetes. Diabetologia. 2016:59(21):371-8.
18. von Scholten BJ, Hansen CS, Hasbak P, Kjaer A, Rossing P, Hansen TW. Cardiac autonomic function is associated with the coronary microcirculatory function in patients with type 2 diabetes. Diabetes. 2016;65(10l):3129-38.

19. Mohammedi K, Woodward M, Hirakawa Y, Zoungas S, Colagiuri S, Hamet P, Harrap S, Poulter N, Matthews DR, Marre M, et al. Presentations of major peripheral arterial disease and risk of major outcomes in patients with type 2 diabetes: results from the ADVANCE-ON study. Cardiovasc Diabetol. 2016;15(1I):129.

20. Lee JM, Jung JH, Hwang D, Park J, Fan Y, Na SH, Doh JH, Nam CW, Shin ES, Koo BK. Coronary flow reserve and microcirculatory resistance in patients with intermediate coronary stenosis. J Am Coll Cardiol. 2016;67(10l):1158-69.

21. Low Wang CC, Hess CN, Hiatt WR, Goldfine AB. Clinical update: cardiovascular disease in diabetes mellitus: atherosclerotic cardiovascular disease and heart failure in type 2 diabetes mellitus - mechanisms, management, and clinical considerations. Circulation. 2016;133(24I):2459-502.

22. Reinhard H, Wiinberg N, Hansen PR, Kjaer A, Petersen CL, Winther K, Parving $\mathrm{HH}$, Rossing $\mathrm{P}$, Jacobsen PK. NT-proBNP levels, atherosclerosis and vascular function in asymptomatic type 2 diabetic patients with microalbuminuria: peripheral reactive hyperaemia index but not NT-proBNP is an independent predictor of coronary atherosclerosis. Cardiovasc Diabetol. 2011;10:71

23. Avogaro A, Fadini GP, Sesti G, Bonora E, Del Prato S. Continued efforts to translate diabetes cardiovascular outcome trials into clinical practice. Cardiovasc Diabetol. 2016;15(11):111

24. Brownlee M. The pathobiology of diabetic complications: a unifying mechanism. Diabetes. 2005:54(61):1615-25.

25. Giacco F, Brownlee M. Oxidative stress and diabetic complications. Circ Res. 2010;107(9l):1058-70.

26. Doney AS, Lee S, Leese GP, Morris AD, Palmer CN. Increased cardiovascular morbidity and mortality in type 2 diabetes is associated with the glutathione $S$ transferase theta-null genotype: a Go-DARTS study. Circulation. 2005:111(221):2927-34

27. Higashi Y, Noma K, Yoshizumi M, Kihara Y. Endothelial function and oxidative stress in cardiovascular diseases. Circ J. 2009;73(31):411-8.

28. Rosa CM, Xavier NP, Henrique Campos D, Fernandes AA, Cezar MD, Martinez PF, Cicogna AC, Gimenes C, Gimenes R, Okoshi MP, et al. Diabetes mellitus activates fetal gene program and intensifies cardiac remodeling and oxidative stress in aged spontaneously hypertensive rats. Cardiovasc Diabetol. 2013;12:152

29. Patel H, Chen J, Das KC, Kavdia M. Hyperglycemia induces differential change in oxidative stress at gene expression and functional levels in HUVEC and HMVEC. Cardiovasc Diabetol. 2013;12:142.

30. Odegaard AO, Jacobs DR Jr, Sanchez OA, Goff DC Jr, Reiner AP, Gross MD. Oxidative stress, inflammation, endothelial dysfunction and incidence of type 2 diabetes. Cardiovasc Diabetol. 2016;15:51.

31. Hopps E, Noto D, Caimi G, Averna MR. A novel component of the metabolic syndrome: the oxidative stress. Nutr Metab Cardiovasc Dis. 2010;20(1I):72-7.

32. Fadini GP, Rigato M, Cappellari R, Bonora BM, Avogaro A. Long-term prediction of cardiovascular outcomes by circulating $\mathrm{CD}_{3}{ }^{+}$and $\mathrm{CD}_{3} 4^{+}$ $\mathrm{CD}_{133^{+}}$stem cells in patients with type 2 diabetes. Diabetes Care. 2017:40(1l):125-31.

33. Rigato M, Bittante C, Albiero M, Avogaro A, Fadini GP. Circulating progenitor cell count predicts microvascular outcomes in type 2 diabetic patients. J Clin Endocrinol Metab. 2015;100(7I):2666-72.

34. Oikawa A, Siragusa M, Quaini F, Mangialardi G, Katare RG, Caporali A van Buul JD, van Alphen FP, Graiani G, Spinetti G, et al. Diabetes mellitus induces bone marrow microangiopathy. Arterioscler Thromb Vasc Biol. 2010:30(31):498-508. 